\title{
Optimal Sequential Vector Quantization of Markov Sources
}

\author{
Vivek S. Borkar 12 \\ Dept. of Computer Science and \\ Automation \\ Indian Institute of Science \\ Bangalore 560012, India \\ Email: borkar@csa.iisc.ernet.in
}

\author{
Sanjoy K. Mitter ${ }^{2}$ \\ Laboratory for Information and \\ Decision Systems \\ M.I.T. \\ Cambridge, MA, 02139 \\ Email: mitter@lids.mit.edu
}

\author{
Sekhar C. Tatikonda ${ }^{2}$ \\ Laboratory for Information and \\ Decision Systems \\ M.I.T. \\ Cambridge, MA, 02139 \\ Email: tatikond@mit.edu
}

Abstract - The problem of optimal sequential vector quantization of Markov sources is cast as a stochastic control problem with partial observations and constraints, leading to useful existence results for optimal codes and their characterizations.

\section{INTRODUCTION}

Traditional rate distortion theory resides in the "asymptopia" of arbitrarily long block codes. Nevertheless, there are several situations, notably in control applications, where long block codes imply long delays. Hence there is a need for "real time" rate distortion theory. In this paper, we consider sequential vector quantization followed by coding itself as an optimal control process. An important insight gained is the key role played by the process of conditional laws of the source given its encodings. This process is given by an appropriate recursive nonlinear filter. The optimization problem then translates into an appropriate "certainty equivalent" control problem for the latter process.

\section{Sequential Vector Quantization}

We assume a stationary Markov source $X_{n}, n \geq 0$, with transition kernel $p(x, d y)$, which is to be mapped (vector quantized) into a finite subset $A$ of its range $S \subset \mathbb{R}^{d}$. The latter is then encoded into a prescribed discrete codebook $\Sigma$. Letting $l_{A}(\cdot), i_{A}(\cdot)$, denote the respective maps, the encoding of $X_{n+1}$ is $q_{n}=i_{Q_{n}} \circ l_{Q_{n}}\left(X_{n+1}\right)$, where $Q_{n}$ is a finite subset of $S$ chosen based on $q_{i}, i \leq n$. (We impose certain mild technical restrictions on $i_{A}$, see [1] for details.) The problem is to choose $Q_{n}$ at time $n, n \geq 0$, based on $q_{m}, m \leq n$, so as to minimize the average entropy rate

$$
\underset{n \rightarrow \infty}{\limsup } \frac{1}{n} \sum_{m=0}^{n-1} E\left[H\left(q_{m+1} \mid q_{i}, i \leq m\right)\right]
$$

subject to a constraint on the average distortion

$$
\limsup _{n \rightarrow \infty} \frac{1}{n} \sum_{m=0}^{n-1} E\left[\left\|X_{m}-i_{Q_{m-1}}^{-1}\left(q_{m}\right)\right\|^{2}\right] \leq D,
$$

for a prescribed $D>0$. This is the "constrained" version of the sequential rate distortion problem. We also consider the "weighted" version wherein we seek to minimize, for a given $\lambda>0$,

$\underset{n \rightarrow \infty}{\limsup } \frac{1}{n} \sum_{m=0}^{n-1} E\left[H\left(q_{m+1} \mid q_{i}, i \leq m\right)+\lambda\left\|X_{m}-i_{Q_{m-1}}^{-1}\left(q_{m}\right)\right\|^{2}\right]$.

\footnotetext{
${ }^{1}$ This work was supported a Homi Bhabha Fellowship.

${ }^{2}$ This research supported by U.S. Army Grant PAAL03-92-G0115, Center for Intelligent Control Systems.
}

\section{Reduction to Control Problem}

Let $\pi_{n}$ denote the conditional law of $X_{n}$ given $q_{m}, m \leq n$. Then one verifies that

$$
E\left[H\left(q_{m+1} \mid q_{i}, i \leq m\right)\right]=E\left[k\left(\pi_{n}, Q_{n}\right)\right]
$$

where

$$
\begin{gathered}
k(\pi, A)=-\sum_{a} h_{a}(\pi, A) \log h_{a}(\pi, A), \\
h_{a}(\pi, A)=\int \pi(d x) \int p(x, d y) I_{\left\{i_{A} \circ l_{A}(y)=a\right\}},
\end{gathered}
$$

( $I$ denotes the indicator function) and

$$
E\left[\left\|X_{n}-i_{Q_{m-1}}^{-1}\left(q_{m}\right)\right\|^{2}\right]=E\left[g\left(\pi_{n}, Q_{n}\right)\right]
$$

where

$$
g(\pi, A)=\int \pi(d x) \int p(x, d y)\left\|y-l_{A}(y)\right\|^{2} .
$$

The problem then reduces to that of optimally controlling the nonlinear filter

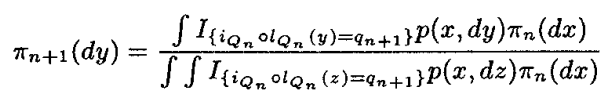

so as to minimize either

$$
\limsup _{n \rightarrow \infty} \frac{1}{n} \sum_{m=0}^{n-1} E\left[k\left(\pi_{m}, Q_{m}\right)\right]
$$

subject to

$$
\limsup _{n \rightarrow \infty} \frac{1}{n} \sum_{m=0}^{n-1} E\left[g\left(\pi_{m}, Q_{m}\right)\right] \leq D,
$$

or that of minimizing

$$
\limsup _{n \rightarrow \infty} \frac{1}{n} \sum_{m=0}^{n-1} E\left[k\left(\pi_{m}, Q_{m}\right)+\lambda g\left(\pi_{m}, Q_{m}\right)\right] .
$$

These can be analyzed by stochastic control techniques to yield the existence of a (possibly randomized) optimal vector quantization-encoder scheme and in the weighted case, a dynamic programming characterization of the optimal scheme. Further research includes using approximate dynamic programming techniques for developing these coding schemes and applications to control problems under communication constraints [2].

\section{REFERENCES}

[1] V.S. Borkar, S. K. Mitter, S. Tatikonda, "Optimal Sequential Vector Quantization of Markov Sources," MIT LIDS report LIDS-P-2395, August 1997, submitted.

[2] V.S. Borkar, S. K. Mitter, S. Tatikonda, "Markov Control Problems Under Communication Constraints," submitted. 